\title{
Risk of dengue occurrence based on the capture of gravid Aedes aegypti females using MosquiTRAP
}

\author{
Lizandra Makowski Steffler, Letícia Silva Marteis, Silvio Santana Dolabella, \\ Sócrates Cabral de Holanda Cavalcanti, Roseli La Corte dos Santos/ ${ }^{+}$
}

Centro de Ciências Biológicas e da Saúde, Universidade Federal de Sergipe, São Cristóvão, SE, Brasil

We assessed the risk classification of dengue fever based on the capture of Aedes aegypti adults using MosquiTRAP, a type of sticky trap, in comparison with traditional larval infestation indices. A total of 27 MosquiTRAPS were installed, with one trap per block, and were inspected weekly between November 2008-February 2009. Infestation baseline data were obtained from a survey conducted prior to trap installation. The index generated by MosquiTRAP and house index (HI) classified the area "in alert situation". The set for risk of dengue occurrence proposed by the use of MosquiTRAP classify areas in the same way of the traditional HI.

Key words: Aedes aegypti - MosquiTRAP - infestation - vector control - Sergipe

Dengue is a major public health problem in Brazil. Frequent epidemics have occurred over time, mainly in the Southeast and Northeast Regions, although the incidence rates are similar in epidemic years in all regions of the country, sometimes exceeding 300 cases $/ 100,000$ habitants (MS 2010). The main indicator of Aedes aegypti infestation that is applied by the National Program for Dengue Control in Brazil is the rate of house index (HI). Due to logistical difficulties and the delay in obtaining data, the Ministry of Health implemented a rapid survey index [Ae. aegypti Infestation Index Rapid Survey (LIRAa)] (Coelho et al. 2008), which is a simplified method of sampling conducted to provide current information on Ae. aegypti infestation. Indices based on immature forms of the vector have been found to be mostly inadequate for the prediction of virus transmission (Focks 2003). Similarly, when specifically assessing the LIRAa, Coelho et al. (2008) observed that it was not a reliable predictor of the incidence of dengue.

Indicators based on traps for adult insects represent a more reliable alternative to larval indices because they estimate the mosquito density associated with the vectorial capacity and the risk of disease transmission. Among the several traps developed to monitor or to control the population of adult mosquitoes, MosquiTRAP can capture gravid females of Ae. aegypti and has shown positive results (Fávaro et al. 2006, 2008, Gama et al. 2007, Lourenço-de-Oliveira et al. 2008, Maciel-de-Freitas et al. 2008, Honório et al. 2009). Regardless of the technique used to estimate the vector infestation, measurement of epidemiological risk indicators is a challenge for

Financial support: CNPq (410542/2006-7), UFS (to LMS)

+ Corresponding author: rlacorte@ufs.br

Received 2 September 2010

Accepted 26 January 2011 entomological surveillance. Eiras and Resende (2009) proposed the Mean Index of Ae. aegypti Females (IMFA) based on MosquiTRAP, which is the ratio between the average number of females caught and the number of traps in the area and the Temporal Mean Index of $A e$. aegypti Females (IMFAt), calculated as the average of the IMFA over four consecutive weeks. In addition, the authors classify the risk for dengue: IMFAt $<0.2$ are riskfree areas, IMFAt between 0.2-0.4 are areas on alert and IMFAt $>0.4$ are areas at risk.

Thus, the purpose of this study was to compare the risk classification proposed by Eiras and Resende (2009) using MosquiTRAP with the current traditional classification of the Ministry of Health based on the index of infestation.

To evaluate these risk classifications, a house-tohouse survey was performed in Porto Dantas $\left(10^{\circ} 52^{\prime} 40^{\prime \prime} \mathrm{S}\right.$ $37^{\circ} 03^{\prime} 42 \mathrm{~W}$ ), a neighbourhood in Aracaju (Northeast Brazil) to collect baseline data. The district is divided by an avenue into two areas: one that is traditionally occupied, with defined and planned blocks of residential buildings, and another, which was recently and irregularly occupied, and is a slum. Most of the inhabitants of the latter area work as recycled garbage pickers and store the collected material in their backyard. All premises were included in the study and were searched for Ae. aegypti larvae and pupae breeding sites, both indoors and outdoors. If the premises were closed at the first visit (18\%), a second one was carried out over the weekend. The larval survey was conducted in November 2008 over two weeks, encompassing 914 (79.4\%) of the 1,150 lots in the neighbourhood, of which 53 (4.6\%) were inspected on weekends. All immature forms of the insect were collected and the species were identified by the Parasitology Laboratory of the Federal University of Sergipe. Following the immature survey, MosquiTRAPs baited with synthetic oviposition attractant were placed in one randomly selected property on each of the 27 blocks. MosquiTRAPs were installed outdoors and monitored weekly over eight weeks in two rounds: 
one between December 2008-February 2009, the dry season, and another between April-June 2009, the rainy season. Species were identified during the trap inspection in the field and later confirmed in the laboratory. After MosquiTRAP inspection, premises were searched for the presence of Ae. aegypti breeding sites.

The larval survey found 1,091 water containers, 14 of which were used as breeding sites by Ae. Aegypti in 12 houses on seven different blocks. Of the 877 immature insects found, 870 larvae and seven pupae were identified. Infestation rates were $\mathrm{HI}=1.3 \%$ and Breteau index $=1.5$, identifying the neighbourhood as on alert according to the threshold $(\mathrm{HI}=1 \%)$ proposed by the Brazilian Ministry of Health (MS 2009). Under these conditions, i.e., when the HI index was approximately $1 \%$, the MosquiTRAPs were $12 \%$ occupied in the first week after the trap was placed and $19.2 \%$ in the third week (Table). This percentage was obtained using one trap per block. Gama et al. (2007) found that approximately $10 \%$ of MosquiTRAPs were positive, whereas the HI was negative. However, in that study, the immature sampling included $10 \%$ of the premises, whereas we covered approximately $80 \%$ of them.

The total IMFA calculated for the dry season resulted in 1.6 mosquitoes per trap. IMFA weekly values ranged between $0.12-0.39$. The IMFAt was 0.20 and 0.25 in the first and second months, respectively, classifying the neighbourhood as "on alert" (Eiras \& Resende 2009). In the wet season, a total IMFA of 0.96 mosquitoes was found per trap. The IMFAt was 0.12 and 0.14 for the first and second months, respectively, thus branding the area a "risk-free" neighbourhood. The classification of risk using IMFAt was similar to the HI used by the Ministry of Health when the survey of breeding sites is done considering the screening of all premises. Although the second survey was carried out in the rainy season, when the vector population was expected to be higher (Dibo et al. 2008), IMFA and IMFAt were lower than in the dry

TABLE

Frequency of MosquiTRAP positivity by week of inspection, Aracaju, Sergipe 2009

\begin{tabular}{lcccc}
\multicolumn{5}{c}{ TABLE } \\
\multicolumn{5}{c}{$\begin{array}{c}\text { Frequency of MosquiTRAP positivity } \\
\text { by week of inspection, Aracaju, Sergipe 2009 }\end{array}$} \\
\hline \multicolumn{4}{c}{ First survey } & \multicolumn{2}{c}{ Second survey } \\
\hline & \multicolumn{4}{c}{ Positives } \\
Weeks & $\mathrm{n}$ & $\mathrm{n}(\%)$ & $\mathrm{n}$ & $\mathrm{n}(\%)$ \\
\hline 1st & 25 & $3(12)$ & 25 & $1(4)$ \\
2nd & 24 & $3(12.5)$ & 22 & $4(18.1)$ \\
3rd & 26 & $5(19.2)$ & 25 & $4(16)$ \\
4th & 22 & $4(18.2)$ & 24 & $3(12.5)$ \\
5th & 25 & $3(12)$ & 24 & $4(16.6)$ \\
6th & 23 & $4(17.4)$ & 24 & $2(8.3)$ \\
7th & 25 & $4(16)$ & 24 & $3(12.5)$ \\
8th & 25 & $4(16)$ & 26 & $2(7.6)$ \\
Mean frequency & - & 15.4 & - & 11.9 \\
\hline
\end{tabular}

period, with values indicative of a risk-free transmission situation. Although these results seem to be inconsistent with the literature, our data can be explained by intense vector control activity, which is usually carried out after an outbreak period. In 2008, 28,543 dengue fever cases were registered in the state of Sergipe, an incidence of 1,430 cases $/ 100,000$ inhabitants (MS 2010). Nevertheless, the "risk-free" classification should be carefully considered because this situation may be ephemeral due to the propitious climatic conditions of the Region and the high number of containers available to mosquitoes. In this way, IMFAt could be a useful tool for evaluating vector control activity. However, for health surveillance, a dynamic system needs to be implemented that can generate new indices in a short time, as proposed by the use of MosquiTRAP and Intelligent Dengue Monitoring technology (Eiras \& Resende 2009).

At the time of the first survey, 13 traps were positive in $48 \%$ of the blocks in the area. At the second timepoint, 12 traps were positive in $44 \%$ of the blocks in the area. The spatial distribution of positive traps in both surveys indicated a non-homogeneous infestation. Traps that were positive more than three times in the second survey were located next to blocks that were positive more than three times in the first one (A in Figure). In addition, positive blocks in the first survey showed eight times more risk of being positive on the next survey [odds ratio $(\mathrm{OR})=$ $8.25(1.45 ; 46.86), p=0.034]$, in spite of the routine mosquito control activities being conducted at the time of the study, including the use of temephos. There was a spatial coincidence between traps that were positive three or more times in the surveys and the blocks that registered the presence of immature insects (B in Figure). However, we did not find positive MosquiTRAPs in blocks that were positive in the larval survey [OR $=1.63(0.29$; 9.26), $\mathrm{p}>0.05]$. This analysis was conducted only for the first survey due to its chronological proximity to the immature evaluation. The persistence of infested areas

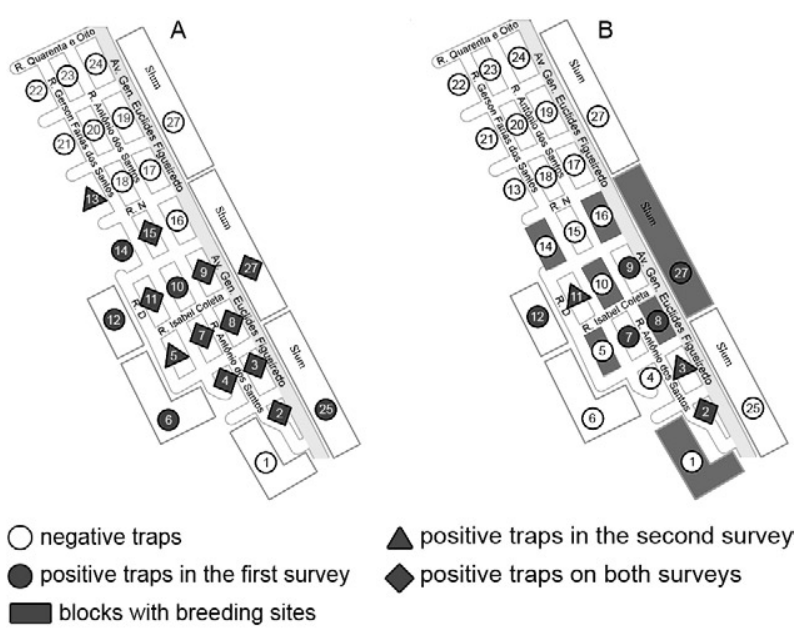

Spatial distribution of MosquiTRAP and breeding sites. A: positive traps on first, second and both surveys; B: at least three times positive traps on first, second and both surveys and blocks with breeding sites. 
or premises and the concentration of breeding sites in a few places has already been recognised (Tun-Lin et al. 1995, Mondini et al. 2005, Honório et al. 2009). In our case, despite the availability of breeding sites across the neighbourhood, the presence of mosquitoes was persistently concentrated in one area of Porto Dantas. This area may represent the hot spots defined by Barrera et al. (2000), from which mosquitoes spread to all districts during favourable epidemic periods, either by climatic conditions or by failing mosquito control measures. The identification of such spreading sites demands direct intervention, aimed at solving either environmental or human problems that contribute to the maintenance of a hot spot. The presence of such persistent infested premises indicates a failure in the application of larvicidal strategy to every permanent container in the area.

In spite of the fact that time series studies with different infestation levels and risk of dengue incidence still need to be performed, our results show that MosquiTRAP and IMFAt are good tools for evaluating vector control activities and mapping vector distribution. However, if MosquiTRAP is more sensitive than the immature survey, as we have shown, a new index or range of risk should be proposed.

\section{REFERENCES}

Barrera R, Delgado N, Jiménez M, Villalobos I, Romero I 2000. Estratificación de una ciudad hiperendémica en dengue hemorrágico. Rev Panam Salud Publica 8: 225-233.

Coelho GE, Burattini MN, Teixeira MG, Coutinho FAB, Massad E 2008. Dynamics of the 2006/2007 dengue outbreak in Brazil. Mem Inst Oswaldo Cruz 103: 535-539.

Dibo MR, Chierotti AP, Ferrari MS, Mendonça AL, Chiaravalloti Neto F 2008. Study of the relationship between Aedes (Stegomyia) aegypti egg and adult densities, dengue fever and climate in Mirassol, state of São Paulo, Brazil. Mem Inst Oswaldo Cruz 103: $554-560$.

Eiras AE, Resende MC 2009. Preliminary evaluation of the "DengueMI" technology for Aedes aegypti monitoring and control. Cad Saude Publica 25 (Suppl. 1): S45-S58.
Fávaro EA, Dibo MR, Mondini A, Ferreira AC, Barbosa AA, Eiras AE, Barata EA, Chiaravalloti-Neto F 2006. Physiological state of $\mathrm{Ae}$ des (Stegomyia) aegypti mosquitoes captured with MosquiTraps in Mirassol, São Paulo, Brazil. J Vector Ecol 31: 285-291.

Fávaro EA, Mondini A, Dibo MR, Barbosa AA, Eiras AE, Neto FC 2008. Assessment of entomological indicators of Aedes aegypti (L.) from adult and egg collections in São Paulo, Brazil. $J$ Vector Ecol 33: 8-16.

Focks DA 2003. A review of entomological sampling methods and indicators for dengue vectors, TDR/IDE/ Den/03.1, World Health Organization, Geneva, $40 \mathrm{pp}$.

Gama RA, Silva EM, Silva IM, Resende MC, Eiras AE 2007. Evaluation of the sticky MosquiTRAP for detecting Aedes (Stegomyia) aegypti (L.) (Diptera: Culicidae) during the dry season in Belo Horizonte, Minas Gerais, Brazil. Neotrop Entomol 36: 294-302.

Honório NA, Codeço CT, Alves FC, Magalhães MA, Lourenço-deOliveira R 2009. Temporal distribution of Aedes aegypti in different districts of Rio de Janeiro, Brazil, measured by two types of traps. J Med Entomol 46: 1001-1014.

Lourenço-de-Oliveira R, Lima JB, Peres R, Alves F da C, Eiras AE, Codeço CT 2008. Comparison of different uses of adult traps and ovitraps for assessing dengue vector infestation in endemic areas. J Am Mosq Control Assoc 24: 387-392.

Maciel-de-Freitas R, Peres RC, Alves F, Brandolini MB 2008. Mosquito traps designed to capture Aedes aegypti (Diptera: Culicidae) females: preliminary comparison of Adultrap, MosquiTRAP and backpack aspirator efficiency in a dengue-endemic area of Brazil. Mem Inst Oswaldo Cruz 103: 602-605.

Mondini A, Chiaravalloti Neto F, Sanches MGY, Lopes JCC 2005. Análise espacial da transmissão de dengue em cidade de porte médio do interior paulista. Rev Saude Publica 39: 444-451.

MS - Ministério da Saúde, Brasil 2009. Diretrizes Nacionais para a Prevenção e Controle de Epidemias de Dengue, Secretaria de Vigilância em Saúde, Departamento de Vigilância Epidemiológica, MS, Brasília, 75 pp.

MS - Ministério da Saúde, Brasil 2010. Doenças de A a Z: dengue/ tabelas [database on the internet]. Brasilia; 2010 [cited 2010 Nov 23]. Available from: http://portal.saude.gov.br/portal/saude/ profissional/area.cfm?id_area $=1525$.

Tun-Lin W, Kay BH, Barnes A 1995. Understanding productivity, a key to Aedes aegypti surveillance. Am J Trop Med Hyg 53: 595-601. 